\title{
Two regimes in the magnetic field response of superconducting $\mathrm{MgB}_{2}$
}

\author{
A. Kohen*, F. Giubileo, Th. Proslier*, F. Bobba, Y. Noat*, A. \\ Troianovski*, A. M. Cucolo, W. Sacks*, J. Klein*, D. Roditchev* \\ Physics Dept. and INFM-SUPERMAT Lab., University of Salerno, via S. Allende, 84081 Baronissi (SA), Italy \\ ${ }^{*}$ Groupe de Physique des Solides, Universités Paris 6 et 7, C.N.R.S. (UMR 75 88), 75015 Paris, France \\ N. Zhigadlo, S.M. Kazakov, J. Karpinski \\ Solid State Physics Laboratory, ETH Zurich, CH-8093 Zurich, Switzerland
}

(Dated: November 19, 2018)

\begin{abstract}
Using Scanning Tunneling Microscope at low temperature we explore the superconducting phase diagram in the $\pi$-band of the two-band superconductor $\mathrm{MgB}_{2}$. In this band the peculiar shape of the local tunneling spectra and their dynamics in the magnetic field reveal the complex character of the quasiparticle density of states (DOS). The gap in the DOS is first rapidly filled with states in raising the magnetic field up to $0.5 \mathrm{~T}$ and then slowly approaches the normal state value : The gap is observed up to $2 \mathrm{~T}$. Such a change in the DOS dynamics suggests the existence of two terms in the DOS of the $\pi$-band: a first one, reflecting an intrinsic superconductivity in the band and a second one, originating from an inter-band coupling to the $\sigma$-band. Our findings allow a deeper understanding of the unique phase diagram of $\mathrm{MgB}_{2}$.
\end{abstract}

The discovery in January 2001 of superconductivity at $39 \mathrm{~K}$ in the well-known binary compound $\mathrm{MgB}_{2}[1]$ has not only opened a new way for potential applications of superconductors but also offered to the scientific community an unexpected field of fundamental research. A huge effort was very soon rewarded: specific heat 2], tunneling [3, 4, 5] and point-contact spectroscopy (PCS) 6, 7] experiments along with the theoretical predictions 8, 9] have shown $\mathrm{MgB}_{2}$ to be a very unusual superconductor in which two electronic bands contribute to the superconductivity in a different way. It was established that in the superconducting state two gaps open at the Fermi level: a leading one, of about $\sim 6-7 \mathrm{meV}$ in the two-dimensional $\sigma$-band and a weak one, of $\sim 2-3 \mathrm{meV}$ in the three-dimensional $\pi$-band.

Since then, the effect was extensively studied in a large number of experimental and theoretical works (for a review see [10]). It was shown that the superconducting gaps are strongly coupled: Both close at the same critical temperature of $39 \mathrm{~K}$ [4]. Thus, in $\mathrm{MgB}_{2}$ superconductivity is not described by two independent condensates of Cooper pairs but rather by a single condensate of a complex nature.

Various studies revealed a peculiar magnetic field response of $\mathrm{MgB}_{2}$. The study of the vortex lattice by Scanning Tunneling Microscopy/Spectroscopy (STM/STS) 11] evidenced an unusually large size of the $\pi$-band vortex cores. This discovery led the authors to suggest that gap in the $\pi$-band is induced by the intrinsically superconducting $\sigma$ band. Heat capacity measurements have shown an anisotropy for $\mathrm{H} \| c$ and $\mathrm{H} \| a b$ [12]. Remarkably, this anisotropy appears only for $\mathrm{H}>0.5$ Tesla, with a large anisotropy developing at higher fields and leading finally to $\mathrm{H}_{c 2} \sim 3 \mathrm{~T}(\mathrm{H} \| c)$ and $\sim 20 \mathrm{~T}(\mathrm{H} \| a b)$. Neutron diffraction experiments revealed a rotation of the flux lattice by $30^{\circ}$ in a field of $\sim 0.6-0.8 \mathrm{~T}$, occur- ring simultaneously with a sharp drop in the diffraction peak intensity [13]. These results were attributed to the suppression of the superconductivity in the $\pi$-band at low fields. Experimentally, the magnetic field response of the two bands was studied in numerous PCS reports [6, 7, 14, 15], however the $\pi$-band gap could be evaluated with reasonable accuracy only up to $0.5 \mathrm{~T}$. Theoretical works regarding the mixed state in a two-band superconductor 16, 17, 18] have qualitatively explained the above findings as a result of the inter-band coupling. Finally, though many experiments revealed a modification in the superconducting properties at the magnetic field of around 0.5-0.8 T, none of the observed changes were unambiguously linked to the evolution of the principal superconducting parameters such as the superconducting gap(s) and/or the inter-band coupling.

In this Letter we address the question of the magnetic field response of the $\pi$-band by means of tunneling spectroscopy. Using STM/STS we studied c-axis oriented surface of $\mathrm{MgB}_{2}$ single crystals. We found the $\pi$-band DOS to deviate significantly from the BCS shape even at $0 \mathrm{~T}$, and to have a non-trivial field dynamics. We observed two distinct regimes: A rapid evolution at low fields $\mathrm{B} \lesssim 0.4 \mathrm{~T}$ and a slow one at high fields $\mathrm{B} \gtrsim 0.6 \mathrm{~T}$, separated by a crossover region where the DOS remains almost unchanged. A clear gap in the $\pi$-band was still observed at $2 \mathrm{~T}$. Such a dynamics suggests the existence of two terms in the $\pi$-band DOS: A first one, reflecting the contribution to the superconducting gap from the electron-electron interaction via phonons within the $\pi$ band, and a second one, originating from an inter-band coupling to the $\sigma$-band. These two terms behave differently in the magnetic field, the first term almost vanishing in the field of $0.6 \mathrm{~T}$. Our experimental findings show the need for an additional theoretical effort in the case of superconductivity in $\mathrm{MgB}_{2}$. 
Single crystals of $\mathrm{MgB}_{2}$ were grown by a high pressure method described elsewhere 20]. The experiments were carried out on a low temperature Omicron STM at Salerno University and on a home-built STM/STS in Paris. The tunneling junctions were achieved by approaching mechanically cut $\mathrm{Pt} / \mathrm{Ir}$ tips to the c-axis oriented surface of the crystals. Due to the peculiar electronic structure of the material, c-axis tunnelling allows to probe mainly the quasiparticle density of states (DOS) in the $\pi$-band [11, 19]. In such a geometry the $\pi$-band component of the local DOS was studied in magnetic field up to $3 \mathrm{~T}$. The superconducting critical temperature was determined locally by measuring the evolution of the tunneling conductance spectra as a function of temperature, and was found to be $38.5 \mathrm{~K}$.

The field dependence of the tunneling conductance spectra $d I / d V(V)$ in the $\pi$-band was obtained by fixing the STM tip in a selected location of the sample and by continuously measuring local $I-V$ tunnelling curves while sweeping the magnetic field, the field in all measurements being parallel to the c-axis. The samples were cooled in zero magnetic field. At low temperature, the field was slowly increased from zero up to the maximum value and reduced to zero. Following, the same procedure was repeated for the reversed polarity. The field sweep rate was $\sim 0.01-0.05 \mathrm{~T} / \mathrm{min}$ and the acquisition time for a single spectrum was $\sim 10$ msec. During such a short acquisition time the field change of $\Delta B \simeq 10^{-6} \mathrm{~T}$ is, much smaller than the field magnitude $\mathrm{B}$ itself, $\Delta B / B<<1$. Thus, the field may be considered as constant for every single data set. In type II superconductors however, the field sweep induces vortex entry into the sample, which modifies the spectra. Indeed, while the STM tip remains fixed the vortices move and from time to time appear under the tip. Statistically some tunneling spectra reflect the DOS in the vortex core, others correspond the DOS between the vortices. Averaging over a large $\left(10^{2}\right.$ $10^{3}$ ) number of consecutively acquired spectra results in a smooth curve representing the quasiparticle DOS spatially averaged over the vortex unit cell 21]. In Fig.1a we show the dynamics of the tunneling conductance spectra (averaged over the vortex lattice unit cell) as a function of the magnetic field. The spectra are characterized by a gap near zero-bias and by only two of the four coherence peaks at $\pm 3 \mathrm{meV}$, consistent with what is expected for the tunneling to the $\pi$-band and in agreement with previous experimental data [3, 5, 6, 7]. The main effect of the magnetic field on the tunneling DOS in Fig. 1a is the filling with the states inside the gap. The field dynamics of the DOS at the Fermi level is represented by the Zero-Bias Conductance (ZBC) in the tunneling spectra (Fig.1b). The evolution of the $\mathrm{ZBC}$ in the magnetic field is unusual : At low fields, the ZBC rises rapidly and reaches a value of about $80 \%$ of the normal state ZBC already at $0.4 \mathrm{~T}$. At higher fields however, the dynamics drastically changes: the filling of states becomes
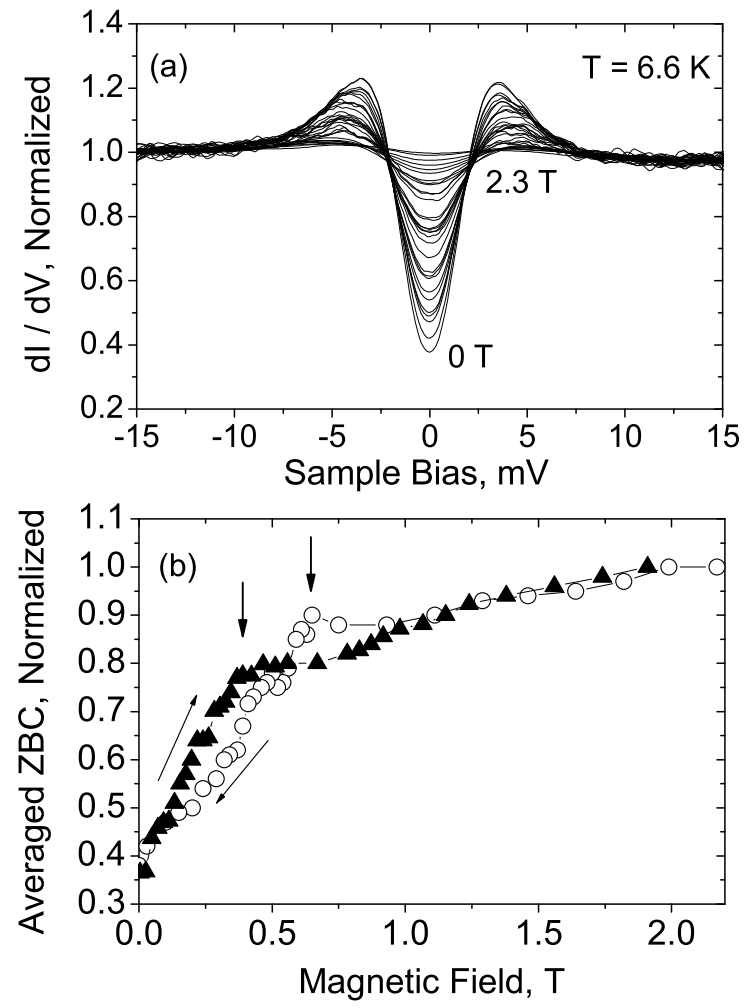

FIG. 1: (a) Evolution of the normalized tunneling conductance spectra in the magnetic fields up to 2.3 Tesla at $\mathrm{T}=6.6$ K. (b) Evolution of the Zero Bias Conductance in increasing (triangles) and decreasing (circles) magnetic field.

much more slower and, even at $2.0 \mathrm{~T}$, it is still possible to distinguish the quasiparticle peaks and a minimum in between in the raw tunneling conductance spectra. At intermediate fields $(0.4<B<0.7 \mathrm{~T})$ there is a crossover region in which the ZBC remains roughly constant. Remarkably, this range matches exactly the range in which a rotation of the vortex lattice was observed [13]. By lowering the field we observe a similar dynamics, though the crossover region is slightly shifted to higher fields. Such a slightly hysteretic behavior indicates different vortex dynamics in increasing and decreasing fields, which may be due to geometrical barriers, vortex pinning, and lattice re-arrangements. In order to follow such an unusual field dynamics we use two different models describing the DOS in the $\pi$-band. The first one considers the superconducting gap to be composed of two terms: a first one originating from the electron-electron interaction via phonons inside the $\pi$-band, and a second one, arising from the superconducting coupling to the $\sigma$-band via phonon exchange. The resulting superconducting gap $\Delta_{\pi}$ is calculated self-consistently 17], but finally, the DOS in the $\pi$-band remains BCS-like:

$$
N_{\pi}\left(E, \Gamma_{\text {Dynes }}\right) \propto \operatorname{Re}\left[\frac{|E|-i \Gamma_{\text {Dynes }}}{\sqrt{\left(|E|-i \Gamma_{\text {Dynes }}\right)^{2}-\Delta_{\pi}^{2}}}\right]
$$



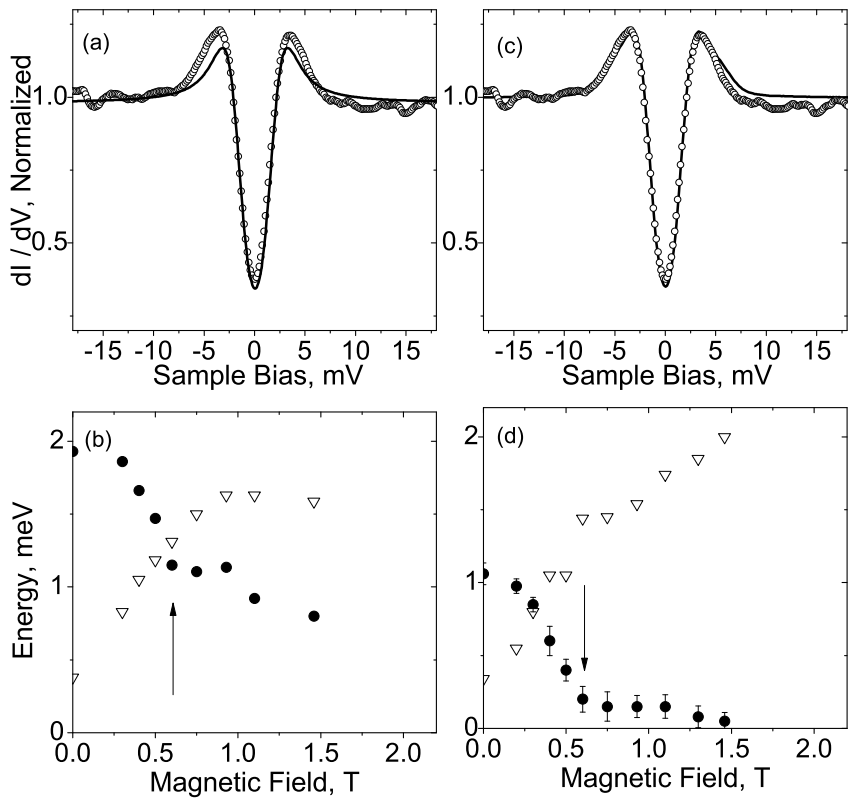

FIG. 2: (a) Zero field spectrum fitted by BCS model (1): $\mathrm{T}=7 \mathrm{~K}, \Delta=1.96 \mathrm{meV}$ and $\Gamma_{\text {Dynes }}=0.32 \mathrm{meV}$. (b) Magnetic field dynamics of superconducting gap (black circles) and pair breaking parameter $\Gamma_{\text {Dynes }}$ (open triangles) extracted from BCS fit of the tunneling spectra. (c) Zero field spectrum fitted using model (2): $\mathrm{T}=6.6 \mathrm{~K}, \Delta_{\pi}^{0}=1.1 \mathrm{meV}, \Delta_{\sigma}^{0}=6.0$ $\mathrm{meV}, \Gamma_{\pi}=1.3 \mathrm{meV}, \Gamma_{\sigma}=0.9 \mathrm{meV}$ and $\Gamma_{\text {Dynes }}=0.3 \mathrm{meV}$. (d) Magnetic field dynamics of superconducting gap (black circles) and pair breaking parameter $\Gamma_{\text {Dynes }}$ (open triangles) extracted from model (2).

In this Dynes formula 22 the pair-breaking parameter $\Gamma$ accounts phenomenologically for the effect of the magnetic field on the DOS.

The second model, in addition, considers the quasiparticle inter-band scattering. To analyze the shape of the DOS in such a case we use, as a first step, two coupled equations proposed by McMillan 23] for the proximity effect in real space. This formalism was already successfully applied in some previous works [24, 25], and it's basic idea is well described in 25]. In the framework of this approach, the DOS in each band deviates from the BCS shape as both $\Delta_{\pi}$ and $\Delta_{\sigma}$ become energy dependent and the apparent gap in the quasiparticle spectrum of the $\pi$-band is larger than the self-consistently calculated superconducting gap, due to the quasiparticle scattering from the $\sigma$-band:

$$
\begin{aligned}
& \Delta_{\pi}(E)=\frac{\Delta_{\pi}^{0}+\frac{\Gamma_{\pi} \Delta_{\sigma}(E)}{\sqrt{\left(\Delta_{\sigma}(E)\right)^{2}-E^{2}}}}{1+\frac{\Gamma_{\pi}}{\sqrt{\left(\Delta_{\sigma}(E)\right)^{2}-E^{2}}}} \\
& \Delta_{\sigma}(E)=\frac{\Delta_{\sigma}^{0}+\frac{\Gamma_{\sigma} \Delta_{\pi}(E)}{\sqrt{\left(\Delta_{\pi}(E)\right)^{2}-E^{2}}}}{1+\frac{\Gamma_{\sigma}}{\sqrt{\left(\Delta_{\pi}(E)\right)^{2}-E^{2}}}}
\end{aligned}
$$

The $\pi$-band DOS is still given by Eq. (1), however $\Delta_{\pi}$ is replaced by $\Delta_{\pi}(E)$, where $\Delta_{\pi}^{0}$ and $\Delta_{\sigma}^{0}$ represent the intrinsic pairing potentials. The effect of finite temperature is accounted for in both models in a standard way by the convolution integral of the DOS (1) with the derivative of the Fermi-Dirac function.

We now focus on the dynamics of the superconducting gap in the magnetic field using the above models. As we will show in both cases the superconducting gap $\Delta_{\pi}$ exhibits a strong change near $0.6 \mathrm{~T}$ (for the decreasing field branch). In Fig.2a we present an example of a BCS fit (1) to the raw tunneling zero field conductance spectrum. We note that this fit fails to reproduce simultaneously the value of the ZBC and the amplitude of the quasiparticle peaks for any field. However, it does allow an estimate for the energy scale of the gap. Fig.2b shows the field dynamics of the main parameters extracted from the BCS fits. In particular, one can see that the superconducting gap exhibits a rapid drop at $0.6 \mathrm{~T}$ while the pair-breaking rises continuously in this field region. In Fig.2c we show a typical fit of the same DOS as in Fig.2a using McMillan model (2). Within this model we find a much better agreement between the experimental data and theoretical curves. Also in this case, the superconducting gap extracted from the fits exhibits a remarkably strong damping at a field around 0.6T (black circles in Fig.2). In all calculations $\Gamma_{\pi}$ and $\Gamma_{\sigma}$ are kept constant since physically the inter band scattering rates should not change significantly in the magnetic field.

The use of McMillan model is not arbitrary but motivated by the fact that experimentally we observe systematic deviations of the tunneling spectra from BCS behaviour. Such discrepancies are better seen in SIS spectra as shown in Fig.3. The spectra were measured at zero field using a superconducting $\mathrm{MgB}_{2}$ tip [4]. In such a SIS geometry the spectroscopic features are enhanced by the convolution of the DOS of two electrodes. One can clearly see a ZB peak characteristic for SIS junctions, much stronger quasiparticle peaks appearing at $\pm 6 \mathrm{meV}$ and not at $\pm 3 \mathrm{meV}$ as in SIN spectra (Fig.1). Remarkably, some additional features, humps and dips are seen on the tails of the quasiparticle peaks. The best fit using BCS DOS is plotted as a thin solid line, the thick one representing the best fit using McMillan approach (2). It is clear that the thick line follows all measured spectroscopic features in finer details. Such an excellent agreement indicates that one should include into the realistic model of the superconductivity in $\mathrm{MgB}_{2}$ a contribution of the quasiparticle inter-band scattering. The values for electron-phonon coupling constants should be probably reconsidered.

We see that the use of both models result in a significant drop of the superconducting gap energy at $0.6 \mathrm{~T}$. This finding is in qualitative agreement with the theoretical prediction 17]. The relative change of the superconducting gap is larger within Mac Millan model (2) since 


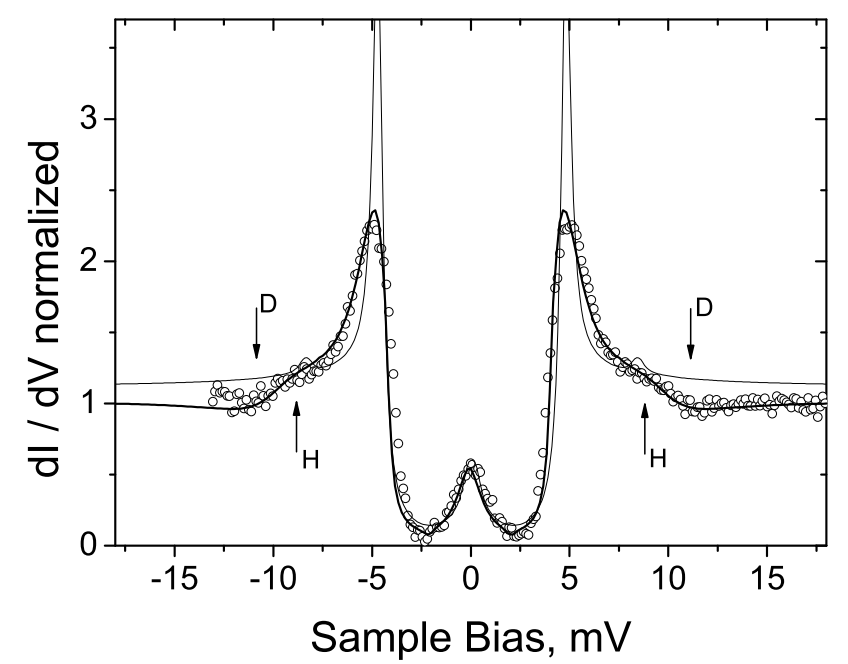

FIG. 3: Circles: Zero field S-I-S tunneling spectrum. Thin solid line: fit using (1) with $\Delta_{\sigma}=6.6 \mathrm{meV}$ (relative weight $2 \%), \Delta_{\pi}(98 \%)=2.35 \mathrm{meV}, \Gamma_{\text {Dynes }}=0.1 \mathrm{meV}, \mathrm{T}=12 \mathrm{~K}$; thick solid line: fit using (2) with $\Delta_{\sigma}^{0}=7.4 \mathrm{meV}, \Delta_{\pi}^{0}=1.0$ $\mathrm{meV}, \Gamma_{\sigma}=2.3 \mathrm{meV}, \Gamma_{\pi}=2.5 \mathrm{meV}, \Gamma_{\text {Dynes }}=0.12 \mathrm{meV}, \mathrm{T}$ $=12 \mathrm{~K}$. Arrows indicate the positions of humps $(\mathrm{H})$ and dips (D) perfectly reproduced by the Mac Millan approach.

there a significant part of the gapped states originates from the quasiparticle scattering from the $\sigma$-band, a process which is field independent. Both models suggest that at the field of $0.6 \mathrm{~T}$ the contribution to the superconductivity from the electron-electron interaction via phonons in the $\pi$-band itself is not efficient anymore. At higher fields the gap in the tunneling DOS survives due to the phonon exchange with $\sigma$-band [26] or due to the both, phonon exchange and quasiparticle inter-band scattering (Mac Millan model (2) ).

We note here that while equation (1) is often used to describe the quasiparticle spectrum in the magnetic field [7], its application is not rigorously justified from a theoretical point of view. Indeed, (1) and (2) initially describe the quasiparticle spectrum in zero field. Even the introduction of $\Gamma_{\text {Dynes }}$ to consider the pair breaking in the magnetic field is not really satisfactory as it is correct only at $\mathrm{B} \sim \mathrm{B}_{c 2}$. We decided to use such a procedure in order to clarify the physical meaning of the field dynamics we experimentally observed, in the lack of a realistic theory describing the DOS in the magnetic field.

In conclusion, in this paper we studied the superconducting $\pi$-band tunneling DOS in single crystals of $\mathrm{MgB}_{2}$ in magnetic field, and we succeeded to observe the superconducting gap up to $2 \mathrm{~T}$. The evolution of the DOS is characterized by two distinct regimes separated by a crossover region. Our results indicate a rapid suppression of the intrinsic term in $\pi$-band superconductivity for $0 \mathrm{~T}$ $<B<0.4 \mathrm{~T}$. At high fields $(0.7 \mathrm{~T}<B<2 \mathrm{~T})$ the superconductivity in the $\pi$-band survives uniquely due to the coupling to the $\sigma$-band. The shape of tunneling spectra suggests an important role played by the quasiparticle inter-band scattering. We think an additional theoretical effort is needed to consider properly the inter-band coupling in the realistic model of the two-band superconductivity in $\mathrm{MgB}_{2}$.

The authors thank A.A. Golubov for useful discussions. This work has been supported by Italian MIUR project "Rientro dei Cervelli" and by the French University Paris $6 \mathrm{PPF}$ project.

[1] J. Nagamatsu et al., Nature 410, 63 (2001)

[2] S.L. Budko et al., Phys. Rev. Lett. 86, 1877 (2001)

[3] F. Giubileo et al., cond-mat/0105146, Europhys. Lett. 58, 764 (2002).

[4] F. Giubileo et al., Phys. Rev. Lett. 87, 177008 (2001).

[5] M. Iavarone et al., Phys. Rev. Lett. 89, 187002 (2002).

[6] P. Szabo et al., Phys. Rev. Lett. 87, 137005 (2001).

[7] R.S. Gonnelli et al., Phys. Rev. Lett. 89, 247004 (2002).

[8] J. Kortus et al., Phys. Rev. Lett. 86, 4656 (2001).

[9] A.Y. Liu et al., Phys. Rev. Lett. 87, 087005 (2001).

[10] Physica C: Superconductivity, Volume 385, Issues 1-2, (1 March 2003), Elsevier

[11] M.R. Eskildsen et al., Phys. Rev. Lett. 89, 187003 (2002).

[12] F. Bouquet et al., Phys. Rev. Lett. 89, 257001 (2002).

[13] R. Cubitt et al., Phys. Rev. Lett. 91, 047002 (2003).

[14] P. Samuely et al., Physica C 385, 244 (2003).

[15] R.S. Gonnelli et al., cond-mat/03081532.

[16] N. Nakai, M. Ichioka, and K. Machida, J. Phys. Soc. Jpn. 71, 23 (2002).

[17] A.E. Koshelev and A.A. Golubov, Phys. Rev. Lett. 90, 177002 (2003).

[18] T. Dahm and N. Schopohl, Phys. Rev. Lett. 91, 187002 (2003).

[19] A. Brinkman et al., Phys. Rev. B 65, 180517 (2002).

[20] J. Karpinski et al., cond-mat/0207264

[21] We suggest this kind of experimental approach as a novel method to study the vortices in superconducting materials in complement to the usual STM/STS (to be published).

[22] R.C. Dynes, V. Narayanamurti, and J. P. Garno, Phys. Rev. Lett. 41, 1509 (1978).

[23] W. L. McMillan, Phys. Rev. 167, 331 (1968).

[24] T. Ekino et al., Phys. Rev. B 67, 094504 (2003).

[25] H. Schmidt et al., Physica C 385, 221 (2003).

[26] H. Suhl, B. T. Matthias, and L. R. Walker, Phys. Rev. Lett. 3, 552 (1959). 